Portland State University

PDXScholar

Physics Faculty Publications and Presentations

Physics

$10-1-2011$

\title{
A simple approach to neutral atom microscopy
}

Philip Witham

Erik J. Sánchez

Follow this and additional works at: https://pdxscholar.library.pdx.edu/phy_fac

Part of the Physics Commons

Let us know how access to this document benefits you.

\section{Citation Details}

Witham, P., and Sánchez, E. (2011). A simple approach to neutral atom microscopy. Review Of Scientific Instruments, 82(10), 103705.

This Article is brought to you for free and open access. It has been accepted for inclusion in Physics Faculty Publications and Presentations by an authorized administrator of PDXScholar. Please contact us if we can make this document more accessible: pdxscholar@pdx.edu. 


\title{
A simple approach to neutral atom microscopy
}

\author{
Philip Witham ${ }^{a)}$ and Erik Sánchez \\ Department of Physics, Portland State University, Portland, Oregon 97223, USA
}

(Received 11 June 2011; accepted 21 September 2011; published online 19 October 2011; publisher error corrected 31 October 2011)

\begin{abstract}
Scanning surfaces using a beam of noncharged atoms or molecules allows for especially nondestructive and low-energy surface imaging, with the potential to obtain new information about surfaces that cannot be easily obtained otherwise. We have developed a new approach, operating with the sample at a close working distance from an aperture, the need for optics to focus the beam is obviated. Compared to more complex approaches, the theoretical performance has no other disadvantage than the short working distance. Resolution of $1.5 \mu \mathrm{m}$ has been achieved, and submicron resolution appears to be practical. Construction of the microscope and results are presented, including first images done in reflection mode, theory for optimization of the design and avenues for future improvement. (c) 2011 American Institute of Physics. [doi:10.1063/1.3650719]
\end{abstract}

\section{INTRODUCTION}

The first image from a neutral atom beam microscope was achieved in 2007. ${ }^{1}$ Such a beam has an energy of under $0.1 \mathrm{eV}$. This is only somewhat higher than the average room temperature thermal energy of gas molecules and of the sample molecules themselves. Yet the de Broglie wavelength is under $1 \AA$, and this energy is usually insufficient to penetrate a single atomic layer. If helium is used, there is additionally no chemical interaction or spin interaction with the sample, and the atoms generally scatter from the sample without adsorption. The possibility exists, therefore, to image the very surface layer of a sample, including any water or contamination layer, and extremely fragile structures without damage. Helium at this energy has a strong scattering interaction with hydrogen, which has a great importance to surface chemistry including catalysts and metallurgy. ${ }^{2,3}$ No coating is needed to image nonconductive samples, and samples need not be sectioned. Highly charged and/or magnetic materials could also be imaged without difficulty.

\section{PREVIOUS EFFORTS AND RESULTS}

In comparison to charged particles, it is difficult to focus particles that have no net charge. Some of the methods that have been successfully used or proposed include those summarized in Table I and the references. Simultaneously obtaining a high beam intensity and a small spot size are required in order to realize a useful microscope.

The best published 2D image resolution obtained so far is on the order of $2 \mu \mathrm{m},{ }^{1}$ and unpublished results ${ }^{13}$ have achieved $0.8 \mu \mathrm{m}$. These images were obtained in transmission mode, and the authors have found no published 2D imaging done in reflection mode. Molecular scanners were reported, ${ }^{14,15}$ but using effusive samples rather than an atom beam.

a) Author to whom correspondence should be addressed. Electronic mail: p.j.witham@ieee.org.

\section{PINHOLE NAM CONCEPT}

The approach presented in this work is analogous to pinhole optics rather than refractive or reflective (focusing) optics. A beam of gas particles is formed by a nozzle and an aperture, operating in high vacuum and free molecular flow (Fig. 1). The beam is directed against a sample located close to the aperture, where the beam has not yet diverged far, for high resolution. The sample (or aperture) is mechanically scanned in two dimensions. A mass filtered detector produces the image (pixel) intensity signal from a gas partial pressure. In reflection mode, contrast is produced at least by differential scattering due to topography. For instance, with the detector optimized for sensing those gas atoms scattered toward one side of the sample, a sample area sloped toward the detector would generally be "brighter." Transmission mode can also be used, but was not the main focus of this experiment.

\section{EXPERIMENTAL MICROSCOPE}

\section{A. Overall design and vacuum system}

A compact neutral atom microscopy (NAM) was built, occupying under $1 \mathrm{~m}^{2}$ of bench space, plus space for electronics and two small mechanical fore pumps (Fig. 2). Two adjacent chambers are evacuated, the "source" and "sample" chambers. A capillary tube injects helium from a regulated pressure helium source through a small free-jet nozzle.

Pressure in the source chamber is maintained between $10^{-2}$ and $10^{-1} \mathrm{~Pa}$ by a $300 \mathrm{l} / \mathrm{s}$ turbo pump (Pfeiffer TPH330 ), operating against the high source gas load. A replaceable aperture separates the source chamber from the sample chamber. The distance between the source and aperture is adjustable from 0 to $250 \mathrm{~mm}$. Pressure in the source chamber is measured by a cold cathode gauge. Foreline pressure is measured by thermocouple vacuum and capacitive manometer gauges and is $2-18 \mathrm{~Pa}$ due to the gas load.

The sample chamber is separately pumped by a smaller turbo pump pair, in series (Leybold TMP 50) and a second mechanical fore pump. This combination was necessary 
TABLE I. Some focusing methods and notes.

\begin{tabular}{|c|c|c|c|}
\hline & Beam flow & Spot size $(\mu \mathrm{m})$ & References \\
\hline $\begin{array}{l}\text { Scattering (reflection) from an etched silicon hologram. A small fraction of the incoming } \\
\text { atoms add to a focused peak }\end{array}$ & 28 atoms $/ \mathrm{s}$ & 100 & 4 \\
\hline Fresnel zone plates in transmission mode. & 530 counts $/ \mathrm{s}$ & 2.0 & $1,5,26$ \\
\hline $\begin{array}{l}\text { Evanescent wave mirrors using laser generated fields near surfaces, possible for very cold } \\
\text { atoms, very shallow angles, and/or extremely smooth surfaces. }\end{array}$ & $\ldots^{\mathrm{a}}$ & $\ldots^{\mathrm{a}}$ & 6 \\
\hline Mirrors using magnetic fields, presently possible for extremely cold atoms. & NA & NA & 7,8 \\
\hline $\begin{array}{l}\text { Scattering from extremely flat silicon surfaces, bent slightly by electrostatic fields to an } \\
\text { elipsoidally curved mirror. Three percent of atoms reflect specularly. }\end{array}$ & $\ldots^{\mathrm{a}}$ & 29 & 9,10 \\
\hline $\begin{array}{l}\text { Atomically flat quantum-stabilized } \mathrm{Pb} \text { surface mirror: Achieves a good fraction }(>15 \%) \text { of } \\
\text { atoms reflected into the focus }\end{array}$ & $<10^{7}$ counts/s & 1.5 & 11,12 \\
\hline
\end{tabular}

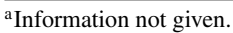

to achieve a sufficiently high compression ratio for the low background helium partial pressure desired. During source operation, He partial pressure in the sample chamber is from $10^{-8}$ to $10^{-7} \mathrm{~Pa}$ and total pressure is between $10^{-6}$ and $10^{-5}$ $\mathrm{Pa}$, as measured by an Inficon Transpector 2 Residual Gas Analyzer (RGA). A netbook style PC performs all of the computing tasks. The beam is projected against the surface of a sample located at $10-500 \mu \mathrm{m}$ from the aperture outlet. The sample is scanned in two dimensions perpendicular to the beam axis by an electromechanical scanner. The RGA, off to one side, produces the partial pressure signal used to form images. An op-amp circuit drives the scanner and a small data acquisition box (Measurement Computing USB1208FS) connects this to the PC. A LabVIEW ${ }^{\mathrm{TM}}$ program was written to produce the scan waveforms and collect the image data over RS232 from the RGA.

The two-dimensional image scan time has been $15 \mathrm{~min}$ to $18 \mathrm{~h}$, and image resolution in pixels from $80^{2}$ to $800^{2}$. The beam intensity appears to be similar to the best achieved to date by neutral atom focusing methods. Long scan times are typical when the system is optimized for best resolution. Note that no focus or astigmatism adjustments are needed, thus the first scan of a sample produces a sharp image and scan time is less of a problem than one might expect from experience with other microscopes.

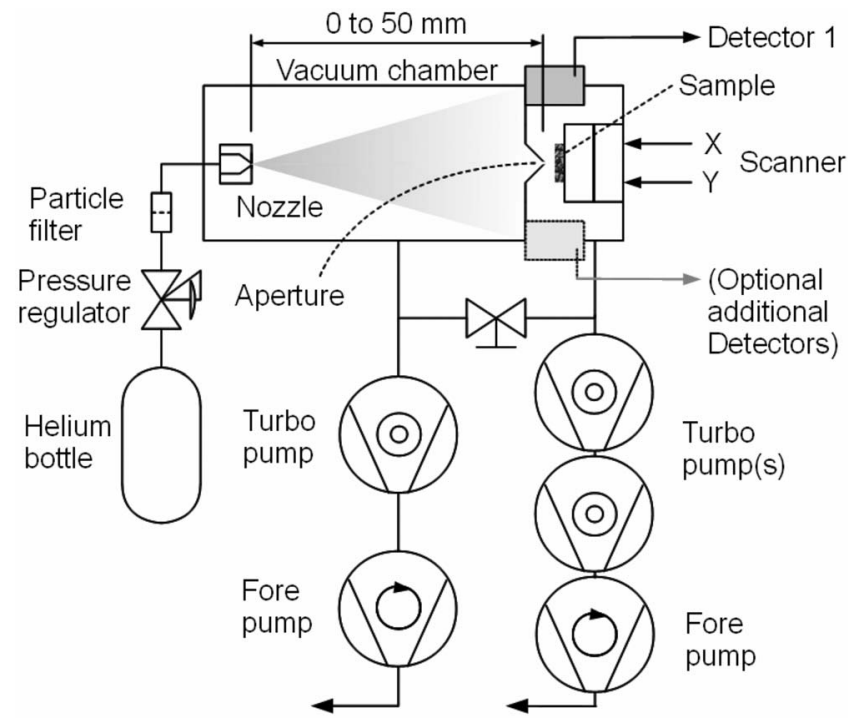

FIG. 1. Conceptual diagram of a pinhole NAM.

\section{B. Source}

The source gas pressure is 0.1-6.9 MPa, depending mainly on the nozzle being used. A $3 \mathrm{~nm}$ filter is used to stop particles that might clog the source nozzle or aperture. Fused silica free jets such as Fig. 3 were pulled from $50 \mu \mathrm{m}$ ID microcapillary tubing using a pipette puller. This is a standard technique mentioned elsewhere. ${ }^{16-18}$ They were selected for tip ID by scanning electron microscope (SEM) imaging. A load lock is provided to exchange the source nozzle, which is mounted on the end of a tube extending into the vacuum through an o-ring seal.

\section{Aperture}

The aperture was formed in a small graphite sheet, $\sim 0.5 \mathrm{~mm}$ diameter by $\sim 1$ um thickness, obtained by pealing a highly oriented pyrolytic graphite monochromator with adhesive tape. This is glued over a $0.35 \mathrm{~mm}$ hole drilled in the tip of a thin aluminum cone, stamped from sheet, about $12 \mathrm{~mm}$ in diameter (Fig. 4(a)). The graphite is then drilled using a Focused Ion Beam system (FEI Co.) to the desired aperture diameter (Fig. 4(b)). The aperture cone is then glued with epoxy to a machined conical aluminum holder. The images of Figs. 7-9 were acquired with a $1 \mu \mathrm{m}$ aperture.

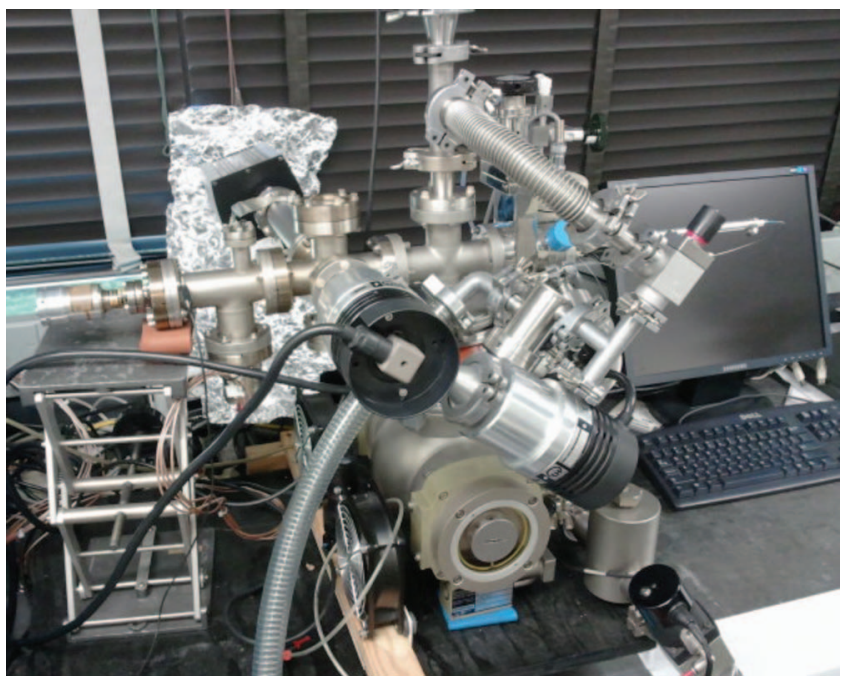

FIG. 2. (Color online) Photograph of the NAM. 


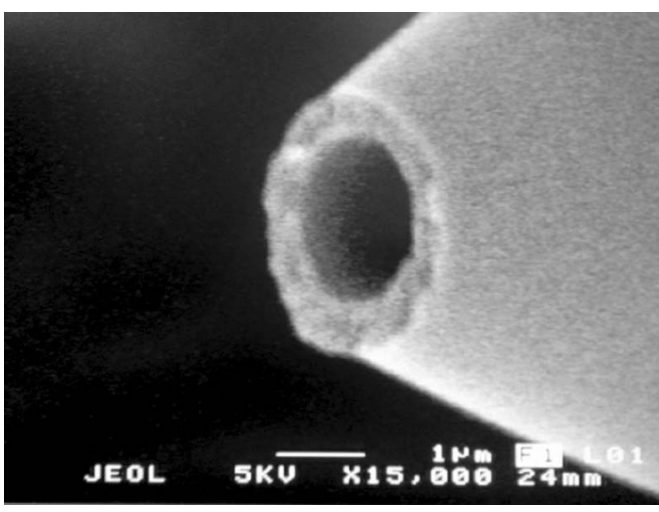

FIG. 3. SEM image of a free-jet source nozzle.

\section{Scanner}

Another advantage a neutral beam is the freedom to incorporate strong magnetic fields in the sample area. Electromechanical scanners can be used. These give the advantages of a wide maximum scan range, simplicity, repeatability, and low hysteresis. For this experiment, the lens focus and tracking actuator of a used CD-ROM drive serves as the scanner. This is a flexure mounted coil assembly with about $1 \mathrm{~mm}$ of maximum range in each of the two axes (Figs. 5(a) and 5(b)). A rare-earth permanent magnet provides the stator field. Approximately $20 \mathrm{~mA}$ at $0.1 \mathrm{~V}$ is required to reach full deflection. The scanner's resonant frequency is on the order of $10-60 \mathrm{~Hz}$ (depending on the mass mounted to it). Either viscous or electronic damping of the scanner is needed to reduce vibration sensitivity at resonance. Electronic damping was provided for the experiment, using a negative resistance driver circuit. A vibration isolation platform is built underneath the microscope, consisting of a sheet of plywood, tennis balls and a 12 in. diameter tire inner tube.

A manual screw positioner was used to adjust the sample $\mathrm{Z}$ approach to the aperture. This is monitored using a stereo optical microscope looking through a vacuum window perpendicular to the beam.

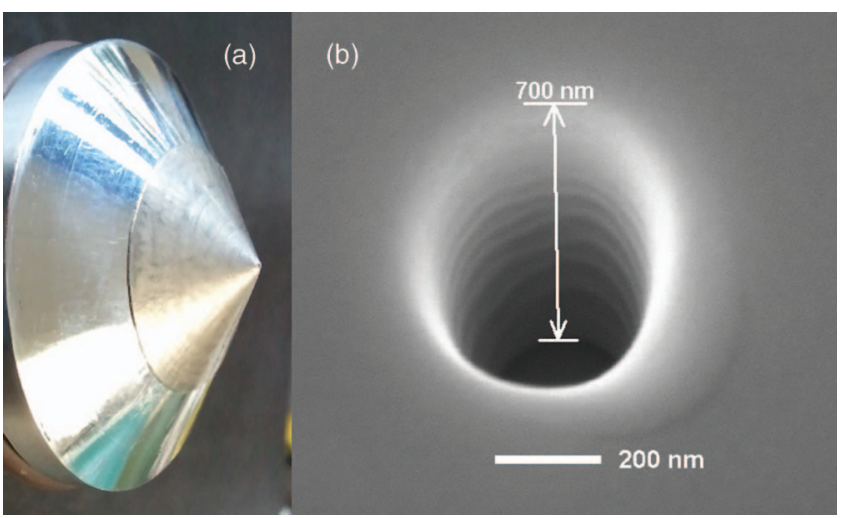

FIG. 4. (Color online) (a) Photograph of the conical aperture holder. (b) SEM image of a $300 \mathrm{~nm}$ ID FIB-drilled aperture in graphite, sample side. (b) is taken at a $15^{\circ}$ angle so the graphite thickness of $\approx 700 \mathrm{~nm}$ can be seen.

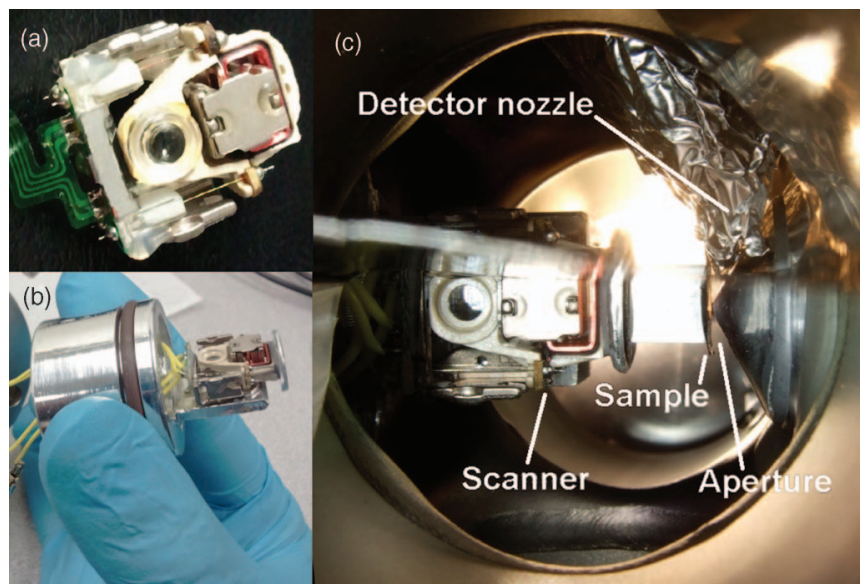

FIG. 5. (Color online) (a) Scanner, (b) scanner assembly, and (c) complete sample chamber arrangement on the right. The detector inlet is towards the top. Note the detector nozzle located above the tip of the aperture cone and sample surface.

\section{E. Detector}

The mass-spectrometer (RGA) samples gas through a nozzle located to one side of the sample area (Fig. 5(c)) and produces a helium partial pressure measurement which is collected in an array. When the full frame has been collected, the software scales the minimum to maximum range of readings to black and white image limits. The range (contrast) from black to white was originally about $6 \%$ of the average pressure after removing noise. In recent scans this has been improved to $25 \%-45 \%$, depending on the sample topography.

The RGA's ability to reject the nearby mass 2 and 3 lines from the mass 4 signal was tested to determine if residual gasses were adding noise to the He signal, particularly hydrogen from water. With the source shut off, the $\mathrm{H}_{2}$ pressure measured $6 \times 10^{-6} \mathrm{~Pa}$ and He pressure measured 1.3 $\times 10^{-11} \mathrm{~Pa}$. With the source turned on, He pressure was 2.8 $\times 10^{-8} \mathrm{~Pa}, \sim 2000 \times$ higher than the background including any RGA selectivity "leakage." Thus ultrahigh vacuum is not needed, due to the mass selectivity of the RGA.

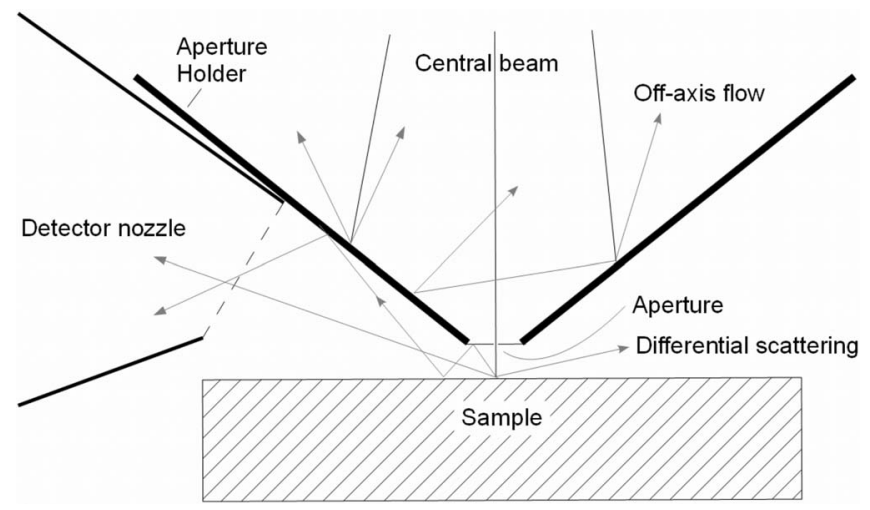

FIG. 6. Aperture and sample area scattering. 


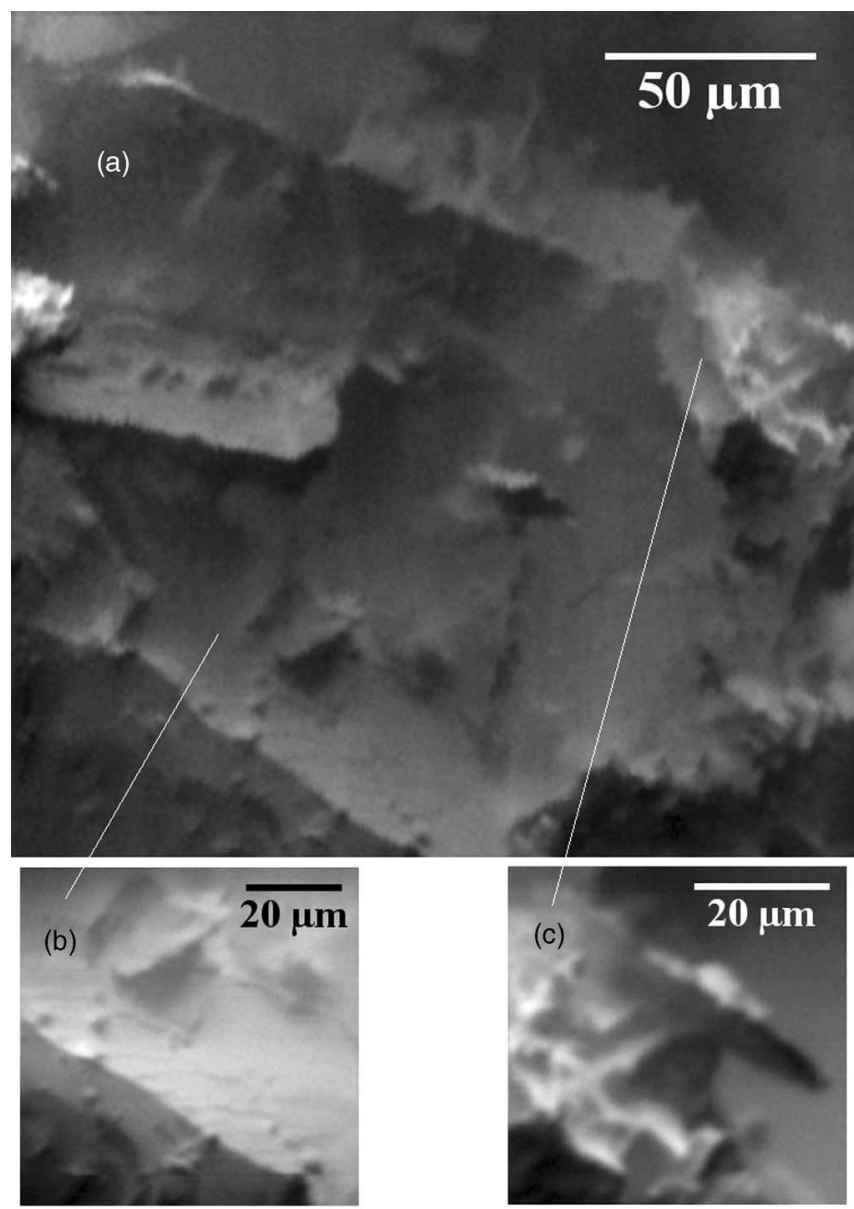

FIG. 7. He scattering images of a crushed high-field NdFeB magnet. (a) An area with fractured planes at three depths, and small particles. (b and c) Magnified areas of the same location.

\section{F. Image results and resolution}

Figures 7-9 had some low pass filtering (Gaussian blur) applied, though without loosing actual resolution. The highest spatial frequency at which sample features can be distinguished from noise is used here as the resolution measurement criteria. For the pollen grain of Fig. 9, the spacing between the closest bright features that can be separated from each other measures $1.5 \mu \mathrm{m}$. Edge sharpness is $1.0 \mu \mathrm{m}$ or better using a 10\%-90\% measurement of adjacent features (Fig. 9(b) and 9(c)). Also apparent is the reduction of sharpness with distance from the aperture, especially in Fig. 8(a), which is a tall magnet particle extending at least $100 \mu \mathrm{m}$ from the background.

All of the images show topological contrast related to the angle of the surface relative to the detector and beam. The detector is toward the top of Fig. 7, the top left of Fig. 8 and the top right of Fig. 9. Its nozzle opening is just above the "horizon" of the sample surfaces. Those edges that face most directly to the detector usually appear brightest. There is also shadowing and diffuse reflection of the "illumination" source (the detector inlet). Figures 7 and 8 demonstrate the microscope's tolerance of high magnetic fields, such an image could not be done by SEM for instance. They are also apparently showing another contrast mechanism, some areas have greater

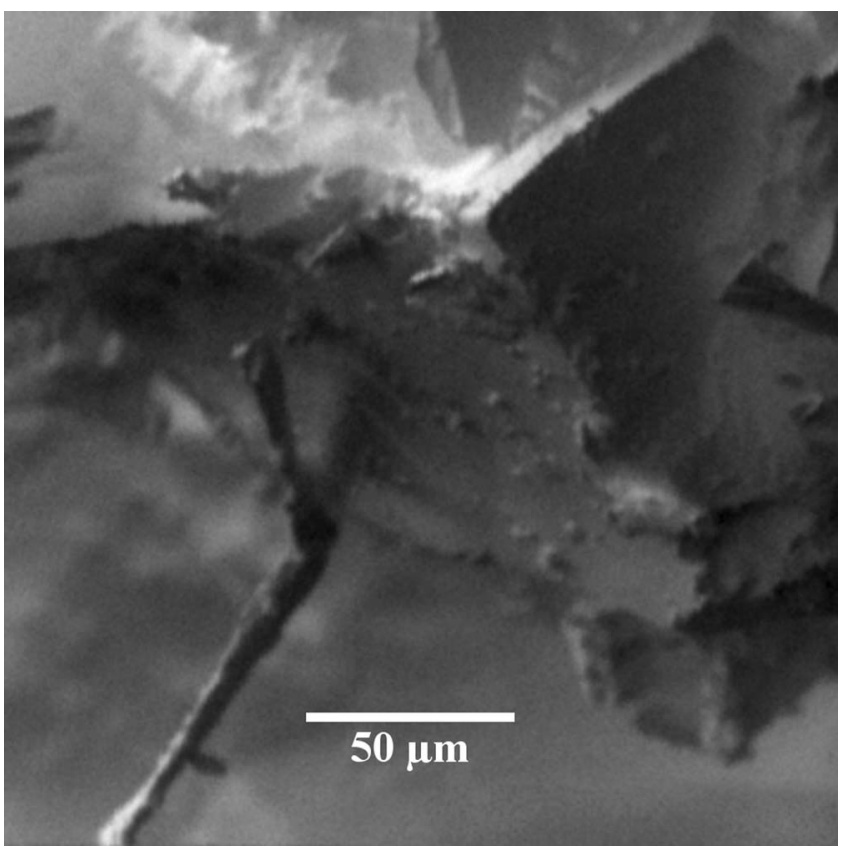

FIG. 8. He scattering images of the same sample as Fig. 7, different location. (a) A large particle extending 100-200 $\mu \mathrm{m}$ from the surface with smaller particles apparently also attached magnetically.

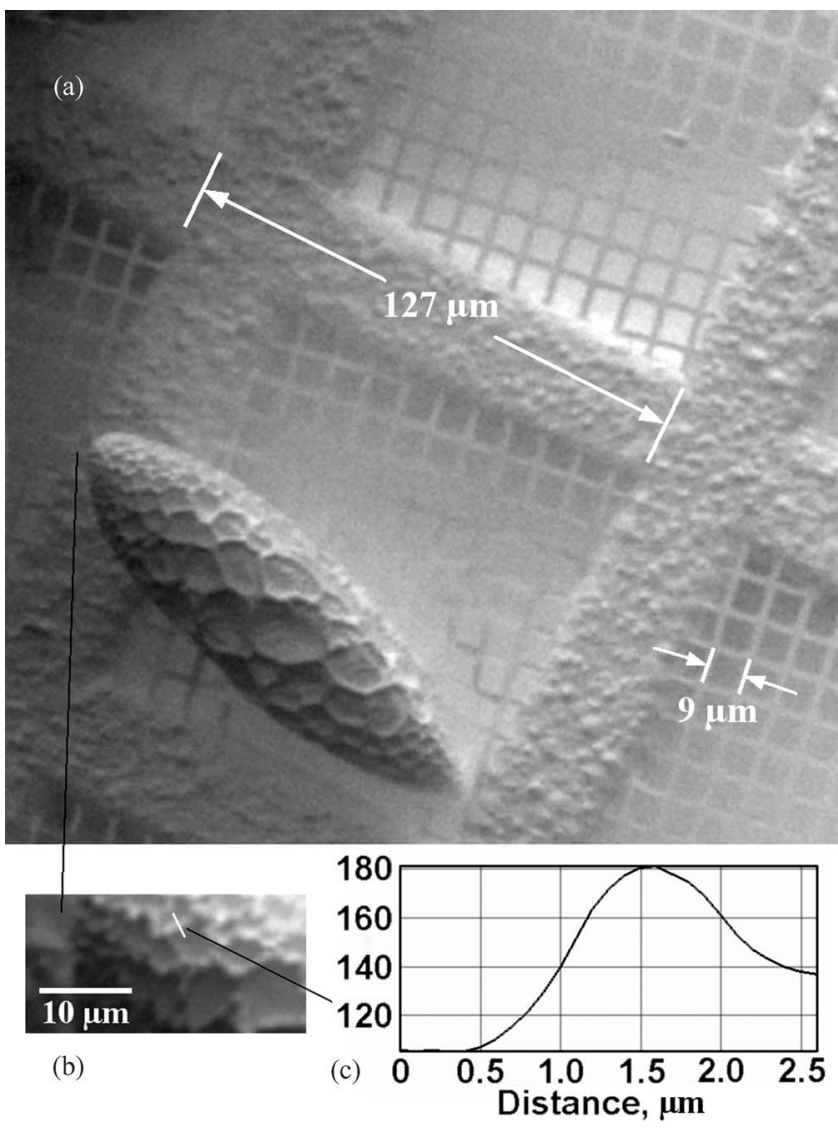

FIG. 9. (a) He scattering image of an uncoated pollen grain on a Quantifoil ${ }^{\mathrm{TM}}$ TEM grid, back side. (b) Magnified area. (c) Line profile taken across linear features in (b), vertical units are grayscale units, where $255=$ white $(\max$. He partial pressure) and $0=$ black (min. pressure). 


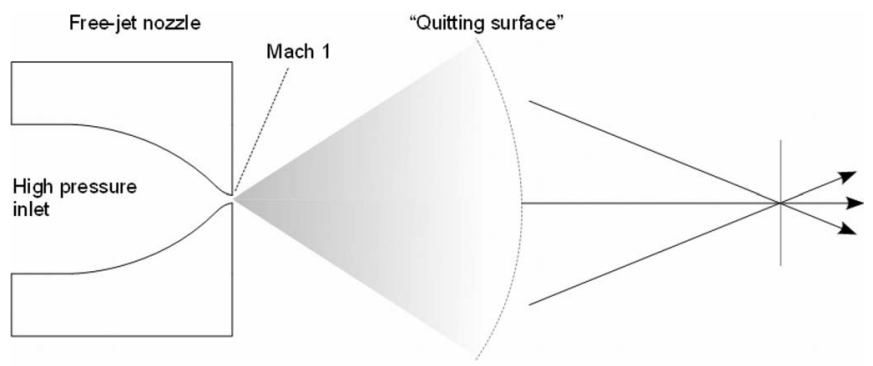

FIG. 10. Diagram of the gas expansion from a free-jet nozzle.

brightness than surrounding areas at the same angle and depth. This is probably due to differences in the degree of specularity (directionality) of the scattering. We speculate that it could be due to surface roughness differences at a scale smaller than the image resolution.

\section{DESIGN THEORY}

Free-jet nozzles are very common in experiments with molecular beams (Fig. 10). Free jets and beam formation are covered well in the literature. ${ }^{16-20}$ Gas is admitted to a vacuum through a small opening. The gas exits at Mach 1 velocity (for that gas at the local temperature) with roughly half of its original pressure, since it cannot obtain a higher velocity than this in a converging space. It then expands into the vacuum, further accelerating, until the pressure has dropped to the point where scattering events between gas molecules are no longer likely. The imaginary more-or-less spherical surface within which the last usual gas-gas scattering events happen is called the "quitting surface." This is the apparent source illumination area from which all beam atoms can be traced (note, however, it is just a useful approximation). From this point on, if the ambient pressure is kept low, the flow is free-molecular.

Often, molecular beam experimenters use additional "skimmer" apertures, chambers, and vacuum pumps to im- prove beam quality. Optimization of the geometry for the experimental microscope showed that this was not necessary, though it is potentially an improvement. A theoretical two-aperture design produced brightness and resolution only somewhat better than the theoretical single-aperture design.

Another difference from a typical molecular beam apparatus is the design of the skimmer, ideally a narrow cone shape pointing upstream into the flow. The purpose of this is to obtain the highest beam quality by minimizing the backscattering of gas into the beam path. In the present use, beam quality is not as critical as clearance on the sample side, and the aperture is on the end of a wide angle cone shape (Fig. 6) pointing downstream (reversed). This is detailed in Sec. B below, including a discussion of the mean free path on the source side as compared to the beam length.

Given the estimate for the quitting surface diameter and the other parameters for the beam geometry (Fig. 11), the approximate spot size can be calculated by

$$
D_{S p o t}=D_{2} \frac{W D}{L_{S e p}}+D_{3} \frac{W D+L_{S e p}}{L_{S e p}} .
$$

$D_{2}$ is determined using Eqs. (2)-(10), which are given below. Alternate system configurations can then be compared for spot size and intensity (Eq. (13)). Examples of optimized configurations determined this way follow in Sec. VI.

The calculated spot size for the current microscope, at the $\sim 50 \mu \mathrm{m}$ working distance of the nearest sample areas, is $1.5 \mu \mathrm{m}$. This matches closely the estimated resolution of the images. Aperture diffraction can be calculated as $a$ $=\operatorname{asin}\left(1.22 \lambda / D_{3}\right)$ where $\lambda=h /\left(m_{h} \times v_{\text {inf }}\right), a$ is the Airy disc half angle and $v_{\text {inf }}$ is the particle velocity from (7) below. This adds $14 \mathrm{~nm}$ to the spot size at $100 \mu \mathrm{m}$ working distance with the current aperture and is not yet significant. An interesting question remaining to be answered is the effect of van der Waals interactions with the aperture walls. Such effects would be angular, hence like the Airy angle, have less effect on the spot size at close working distances to the sample.

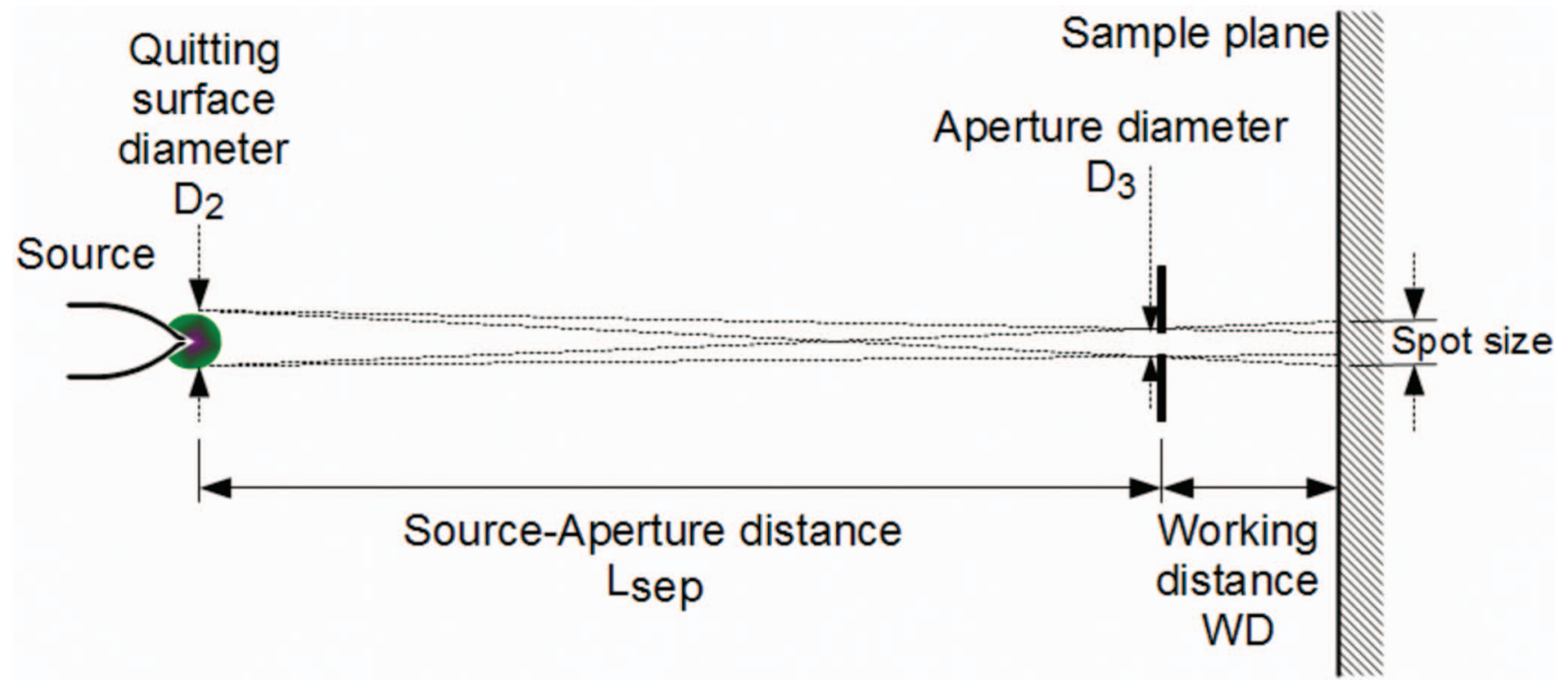

FIG. 11. (Color online) Geometry of the beam. (Not to scale). 
The goals for optimization are to minimize the spot size while maintaining a constant spot intensity ( $n_{\text {beam }}$ in atoms/s) as necessary for a constant $\mathrm{S} / \mathrm{N}$ ratio. This requires obtaining the highest possible beam intensity at the aperture, while minimizing the included angle from the aperture to the quitting surface, minimizing beam divergence.

Some general rules are as follows with equations below. Decreasing the source to aperture distance increases the beam intensity by the inverse square, but increases the beam divergence angle. Increasing the gas flow pushes up the pressure in the source chamber, and at some point causes excessive scattering. But increasing gas flow does not necessarily increase beam intensity since it forces moving the source farther from the aperture for a constant spot size.

\section{A. Source}

Formulae for adiabatic expansion found in Miller ${ }^{17}$ give us the Mach 1 limited conditions at the smallest point in the nozzle. First calculate the throat pressure, $P_{1}$ (in $\mathrm{Pa}$ ),

$$
P_{1}=P_{0}\left(\frac{\gamma-1}{2}+1\right)^{\frac{\gamma}{1-\gamma}},
$$

given the inlet pressure $P_{0}$ and $\gamma$, and the gas ratio of specific heats (1.660 for monatomic gas). The throat temperature $T_{1}$ (in $\mathrm{K}$ ) is calculated,

$$
T_{1}=T_{0}\left(\frac{P_{1}}{P_{0}}\right)^{\frac{\gamma-1}{\gamma}},
$$

from $T_{0}$, the inlet temperature, $v_{1}$, the throat average axial velocity (in $\mathrm{m} / \mathrm{s}$ ) is calculated,

$$
v_{1}=\sqrt{\frac{\gamma k T_{1}}{m_{h}}},
$$

using the Boltzmann constant $k\left(1.381 \times 10^{-23} \mathrm{~J} / \mathrm{K}\right)$ and helium mass $m_{h}(\mathrm{~kg})$. Next, calculate the throat area $A_{1}\left(\mathrm{~m}^{2}\right)$,

$$
A_{1}=\frac{\pi D_{1}^{2}}{4},
$$

given its diameter $D_{1}$ (in $\mathrm{m}$ ). From this the atom flow rate, $n$ (atoms/s) can be calculated,

$$
n=\frac{P_{1} A_{1} v_{1}}{k T_{1}} .
$$

The ultimate axial gas velocity after full expansion into the vacuum, $v_{i n f}$, is

$$
v_{\text {Inf }}=\sqrt{2 \frac{k}{m_{h}}\left(\frac{\gamma}{\gamma-1}\right) T_{0}} .
$$

For example, with helium at $300 \mathrm{~K}$ temperature and $5.5 \mathrm{MPa}$ (800 psia) pressure, and a $2 \mu \mathrm{m}$ diameter nozzle, about 2.4 $\times 10^{18}$ atoms/s exit into the source vacuum chamber at $882 \mathrm{~m} / \mathrm{s}$ axial velocity, $226 \mathrm{~K}$ temperature, 2.7 MPa pressure, and $v_{\text {inf }}$ is $1770 \mathrm{~m} / \mathrm{s}$.

Next, the point where the pressure has dropped to where the mean free path prevents further collisions can be taken as the position of the quitting surface. Using the continuum approximation for the expansion we can obtain a function of Mach number versus distance from the nozzle exit, $M(x)$. Setting the ultimate Mach number $M_{i n f}=M(x)$ we can determine $x$. Following Miller ${ }^{17}$ the formula below combines many details and experimental fits to estimate $S_{i n f}$, the speed ratio for the gas after expansion (the mean velocity divided by the thermal spread of velocities). Helium is assumed from here on,

$$
S_{\text {Inf }}=0.778\left[P_{0} D_{1}\left(9.57 \times 10^{4} \frac{s^{2}}{k g}\left(\frac{K}{T_{0}}\right)^{\frac{4}{3}}\right)\right]^{0.495},
$$

and from this we calculate the Mach number, $M_{i n f}$,

$$
M_{i n f}=\sqrt{\frac{2}{\gamma}} S_{i n f} .
$$

Next, calculate the position $x$ from the nozzle exit where the quitting surface has been reached. To be conservative we can assume that $x$ is equal in all directions (spherical approximation), maximizing $D_{2},\left(D_{2}=2 x\right)$,

$$
D_{2}=2 D_{1}\left(\frac{M_{i n f}}{3.232}\right)^{\frac{1}{\gamma-1}} .
$$

In our example the results are $S_{\text {inf }}=17, M_{\text {inf }}=19$, and $D_{2}=58 \mu \mathrm{m}$. Beam energy is straightforward,

$$
e_{b}=\frac{m_{h} v_{i n f}^{2}}{2},
$$

and equals $65 \mathrm{meV}$ in our example.

An experimental value for the centerline intensity downstream of a free jet is somewhat more than would be expected from a spherical expansion assumption, as detailed in Miller. ${ }^{17}$ This is due to a concentration along the nozzle axis. It is empirically calibrated with a constant " $K_{h}$," equal to 2.0 , and thus $I_{0}$ (in atoms/s $\times$ sr) can be developed from $n$ above,

$$
I_{0}=K_{h} \frac{n}{\pi \mathrm{sr}} .
$$

$I_{0}$ is on the order of $1.5 \times 10^{18}$ atoms $/ \mathrm{s} \times$ sr for the example conditions. Then from the aperture diameter $D_{3}$ and source to aperture distance $L_{\text {sep }}$, the beam flow rate can be predicted

$$
n_{\text {beam }}=I_{0} \frac{\pi}{4}\left(\frac{D_{3}}{L_{\text {sep }}}\right)^{2} \mathrm{sr} .
$$

For the configuration used to capture Figures 7-9 (see Table II), calculated $n_{\text {beam }}$ was approximately $8 \times 10^{10}$ to 10 $\times 10^{10}$ atoms/s. He partial pressure measurements roughly agree with this. Multiplying the atom flow rate by the electron charge $\mathrm{q}$ gives an equivalence to a probe current of $14 \mathrm{nA}$.

This intensity appears to be quite high, though a direct comparison with focusing efforts is difficult. The lack of any loss of the beam to diffuse reflection from a mirror is only one factor in this. Mirror experiments by Barredo et al. ${ }^{11,12}$ indicated a count rate (presumably detector counts) of somewhat under $10^{7}$ per second, but it is difficult to relate this to the atom intensity at a given spot size. One would presume that in time focusing methods can achieve higher inten- 
sities than pinhole optics, but this does not currently appear to be the case.

The actual flow rate of the source is reduced somewhat by viscous effects that depend on the internal size and shape of the nozzle, and these calculations do not predict that. Miller ${ }^{17}$ recommends correcting for this by using a measured flow rate and calculating back to an effective value for $D_{1}$. In that case, it would be necessary to use the corrected $D_{1}$ value consistently (in Eqs. (5), (8) and (10)).

\section{B. Conical aperture}

The aperture design faces several requirements. It must place the aperture hole itself close to the sample. The hole must not scatter the beam by creating a high pressure area, as would occur if it was a long cylinder in shape (hole diameter smaller than the material thickness.) Next, gas atoms scattering from one side the sample should ideally travel to the detector directly, with as few additional scatterings as possible. Each additional scattering event in this pathway will dilute the probability of the atom reaching the detector, and thus reduce the image $\mathrm{S} / \mathrm{N}$ ratio. So optimally, the aperture is at the narrow end of a cone or wedge shape to maximize open area leading from the sample (Fig. 6). However, the source side of the aperture must not concentrate the gas flow excessively, or it would scatter the beam.

Background pressure at the source side produces a random gas particle flow through the aperture. As a result, the beam is superimposed on this "spray" flow. It was found that if the source nozzle is retracted too far, the spray flow exceeds the beam flow through the aperture. This would produce a diffuse image. In addition there is Beer-Lambert law attenuation of the beam, greater scattering with greater beam length. Both of these effects become significant at about the same background pressure.

Thus the source nozzle must be close enough for the beam to dominate. This is easily seen by watching changes in the sample chamber helium partial pressure with changes in the nozzle to aperture distance $\left(L_{\text {sep }}\right)$. Above some distance, the pressure no longer follows an inverse square relationship to the distance, indicating beam flow no longer dominates over nonbeam flow. At high flow rates in the experimental microscope, this occurs at only a few cm distance.

At the reduced flow rates and very close $L_{\text {sep }}$ now used, this is not a problem. This is a Knudsen number question $(\lambda / L)$, with the mean free path being increased during development from $30 \mathrm{~mm}$ to over $100 \mathrm{~mm}$ by reducing the flow rate and $L_{\text {sep }}$ (the distance the gas must traverse to reach the aperture) reduced from $6 \mathrm{~mm}$ to $1 \mathrm{~mm}$. So the Knudsen number increased from 5 to 100 . Knudsen numbers of at least 10 are considered free molecular flow.

The effect of the reflection of the source from the backside of the aperture deserves discussion. Logically, if the flow rate is low enough, the source flow and its reflection superimpose on each other without interaction-gas-gas scattering is not common. At some higher flow rate, the scattered reflection would increase the gas pressure enough to cause excessive gas-gas scattering, and the flow would no longer remain free-molecular. Momentum from the source would begin to push gas toward the aperture and holder, and a standing shock wave might even form. Counter-intuitively, if the source is moved closer-but flow reduced to maintain constant intensity at the aperture - the problem is reduced - the Knudsen number is raised. This question has the same answer as the "spray" flow problem, at a low enough flow rate the Knudsen number is high, and this reflection is not a problem.

\section{Detectors}

Several detector types have been proposed or tried for NAM. Most involve ionizing the gas atoms and then selecting for a particular mass, using a quadrupole mass spectrometer or $90^{\circ}$ magnetic sector. The selected ions are then collected and optionally amplified with an electron multiplier. Mass 4 (helium) has a very low background partial pressure in the vacuum, which allows detection at low partial pressures. Usually an electrospray ionizer with a thermionic emitter is used, and roughly $70 \mathrm{eV}$ electron energy.

The ionizer has limited input area and does not ionize all of the helium atoms entering it. Only a small fraction of the atoms scattered off the sample are detected. Detected ions are neutralized and return to the same gas volume from which they came, and may be detected again. Inherently, this is a partial pressure sensor with some noise level, best considered as an equivalent noise pressure. Since higher microscope resolution requires a smaller aperture area and thus lower beam intensity (gas flow), one always faces a compromise between resolution and noise when selecting the aperture. Lowering the noise level in the detector is crucial.

\section{Detector nozzle}

In reflection mode, image contrast (signal) is created by selecting some general scattering direction from the sample, an included angular area, that will be detected. We can call these atoms "contrast positive." Isolating this subset of the scattered gas with a nozzle is necessary for maximizing the pressure signal. One of the reasons for this is the presence in the sample chamber of gas scattered in the opposite of the chosen "contrast positive" direction ("contrast negative"). These would reduce the contrast if detected. There are also "contrast neutral" atoms scattered in directions roughly perpendicular to the contrast axis, which would only increase the background pressure and noise. Additionally, gas entering the isolated detector volume behind this nozzle can only exit via the inlet area. This results in a decay time constant when connected to the internal vacuum volume of the detector. The open area becomes the apparent illumination source in the image, in a similar way to the effect of the location of the secondary electron detector in SEM. Thus optimizing the location and area of the detector nozzle inlet is important.

\section{Detector noise performance, equivalent area}

To see the ultimate potential of the microscope one has to determine the present detector limitations. Fundamentally, 
a best case detector could count the rate of impingement of (correct mass) gas atoms on the detector nozzle inlet area (n/s) The result is the same if we count the number of atoms in a detector volume, by setting that volume to give a pressure response time constant equal to the sampling period ( $\tau=$ volume/conductance). The Poisson noise that results would have a standard deviation equal to square root ( $\mathrm{n} / \mathrm{sample}$ period). An actual detector will have a higher noise level than this.

It is useful to compare a detector's performance using the standard deviation of its measurements (noise), to that of an ideal detector of some inlet area. To do this, first we start with the rate of particle impingement on an area (based on multiplying the particle velocity by the density of particles). ${ }^{21}$ If we set the impingement rate equal to one per sample period (standard deviation of 1), we obtain a detector equivalent area,

$$
a_{d}=\frac{\sqrt{2 \pi m_{h} k 300 K}}{p_{n} t},
$$

where $p_{n}$ is the noise (standard deviation) equivalent pressure (in $\mathrm{Pa}$ ) and $t$ is the sample period.

For example, some reasonably priced commercial quadrupole mass spectrometers have a specified noise level near $10^{-12} \mathrm{~Pa}$ (measured as the standard deviation in helium partial pressure measurements, using a $1 \mathrm{~s}$ sample period). This is equivalent to an ideal detector sampling an area of only $13 \mu \mathrm{m}^{2}$. The chosen detector nozzle inlet area used to collect Figures $7-9$ was $10 \mathrm{~mm}^{2}$, almost $10^{6}$ greater. This is an estimate of just how much room for improvement there is in the detector. Conventional mass spectrometers have small ionizer inlet areas, and only ionize a few thousandths of a percent of helium atoms. ${ }^{22-24}$ Many things have been tried and at least one achieved a $70 \%$ detection efficiency, ${ }^{23}$ but only for a $2 \mathrm{~mm}$ diameter collimated incoming molecular beam. Nonetheless, that is a substantial improvement over the very good RGA used in this experiment, perhaps $2000 \times$ improvement, based on quoted sensitivities of $2.2 \mathrm{~mA} / \mathrm{Pa}^{23}$ versus the $1.1 \mu \mathrm{A} / \mathrm{Pa}$ specification for the RGA.

Improving the detector is a main variable for improving resolution. Some improvement in the source intensity is possible. A useful figure of merit for the system performance as a whole is obtained by multiplying the detector equivalent area by the source intensity $I_{0}$. The physical limit appears to be at least $10^{6} \times$ better than the current microscope based on the area comparison above, with a practical long-term goal based on known technologies being at least $10^{3} \times$ based on better ionizers ${ }^{23}$ and previously attained source intensities. ${ }^{16-20}$ Resolution should initially improve between the fourth root and the square root of this figure, depending on working distance.

\section{CONCLUSION}

\section{A. Optimization results}

Estimates of potential spot size improvement are given in Table II, using the scan times and $\mathrm{S} / \mathrm{N}$ ratios that produced Fig. 7-9 images. Several configurations are shown, of decreasing working distance. This table assumes that we are reducing the image dimensions in proportion to spot size (in other words, increased magnification). The accuracy of the
TABLE II. Potential future development ( $n_{\text {beam }}$ held constant).

\begin{tabular}{lcccc}
\hline \hline Example & Previous & $\begin{array}{c}\text { Current } \\
\text { (Fig. 7-9) }\end{array}$ & Future 1 & Future 2 \\
\hline Detector improvement & None & None & None & $10 \times$ \\
Working distance $W D(\mu \mathrm{m})$ & 100 & 50 & 33 & 10 \\
Source pressure $(\mathrm{MPa})$ & 2.8 & 0.14 & 6.9 & 6.9 \\
Source throat $D_{1}(\mu \mathrm{m})$ & 3 & 3.5 & 0.140 & 0.063 \\
Aperture $D_{3}(\mu \mathrm{m})$ & 1 & 1 & 0.34 & 0.08 \\
$L_{\text {sep }}(\mathrm{mm})$ & 4 & 1 & 0.100 & 0.033 \\
Spot size $(\mu \mathrm{m})$ & 2.8 & 1.5 & 0.67 & 0.17 \\
\hline \hline
\end{tabular}

predicted spot size depends on Eqs. (10) and (13) remaining accurate to very small values of $D_{1}$, which we have not yet proven in experiment.

\section{B. Future potential and improvements}

The source centerline intensity might be increased by constructing a converging-diverging (de Laval) nozzle. This has been mentioned elsewhere. ${ }^{16,25}$ Such nozzles have been tried here but testing of this technique is not conclusive.

With construction improvements, we could reduce the working distance until reaching the limit of the optics used to watch the $\mathrm{z}$ approach, perhaps under $10 \mu \mathrm{m}$. One could go farther than this using other techniques to sense how close the sample is to contact, but at the same time several other difficulties occur. One is reduced depth of field, which would make relatively flat samples a requirement. Another difficulty is the need for an ever sharper and thinner cone-shaped aperture holder, and more rigid (or active) control of the position.

Using two detectors on opposite sides of the sample may produce an improvement in $\mathrm{S} / \mathrm{N}$ ratio when operated as a differential pair. More detectors (and detector nozzles) radially around the sample would allow simultaneous collection of images from a variety of illumination directions.

The angle of the scanner (and sample), and the position of the detector nozzle inlet could be optimized to maximize the brightness of specular reflections from the sample surface. This would increase the contrast produced by differences in the specularity of sample areas, while perhaps reducing the effect of topography. This may be of use for observing surface characteristics.

Transmission mode could be implemented by putting the detector (nozzle) behind the sample with all else being the same as reflection mode. Another configuration would place the sample where the aperture is located in the previous variants, and the aperture where the sample was, at the entrance to the detector nozzle.

\section{ACKNOWLEDGMENTS}

Many thanks to Professor Erik Sánchez for the use of his laboratory, vacuum equipment, and advice. Everett Lapp for donated RGAs, pumps, and vacuum hardware, Professor Rolf Köenenkamp for his reviews and insights, Mike DeArmond for FIB help, review and help in general, and Dr. Derek Nowak for his LABVIEW ${ }^{\mathrm{TM}}$ expertise and review. 


\section{APPENDIX: NAMING CONVENTION}

Several names have been used for this type of instrument, including Atomic deBroglie Microscope, Helium Microscope, and Scanning Helium Microscope (SHeM). The use of helium is not required, and helium in the name has caused confusion with helium ion microscopes. As mention of deBroglie's name is not descriptive, neutral atom microscope (NAM) is preferred by the authors even though molecular gasses could also be used.

${ }^{1}$ M. Koch, S. Rehbein, G. Schmahl, T. Reisinger, G. Bracco, W. E. Ernst, and B. Holst., J. Microsc. 229, 1 (2008).

${ }^{2}$ D. A. MacLaren, B. Holst, D. J. Riley, and W. Allison, Surf. Rev. Lett. 10, 249 (2003).

${ }^{3}$ See http://www-sp.phy.cam.ac.uk/research/mirror.php3 for more information.

${ }^{4}$ F. Shimizu, Phys. Rev. Lett. 88(12), 123201 (2002).

${ }^{5}$ R. B. Doak, R. E. Grisenti, S. Rehbein, G. Schmahl, J. P. Toennies, and C. Wöll, Phys. Rev. Lett. 83, 4229 (1999).

${ }^{6}$ V. I. Balykin, V. S. Letokhov, Y. B. Ovchinnikov, and A. I. Sidorov, Phys. Rev. Lett. 60, 2137 (1988)

${ }^{7}$ C. V. Saba, P. A. Barton, M. G. Boshier, I. G. Hughes, P. Rosenbusch, B. E. Sauer, and E. A. Hinds, Phys. Rev. Lett. 82, 468 (1999).

${ }^{8}$ D. C. Lau, A. I. Sidorov, G. I. Opat, R. J. McLean, W. J. Rowlands and P. Hannaford, Eur. Phys. J. D 5, 193 (1999).

${ }^{9}$ B. Holst and W. Allison, Nature 390, 244 (1997).

${ }^{10}$ K. Fladischer, H. Reingruber, T. Reisinger, V. Mayrhofer, W. E. Ernst, A. E. Ross, D. A. MacLaren, W. Allison, D. Litwin, J. Galas, S. Sitarek,
P. Nieto, D. Barredo, D. Farías, R. Miranda, B. Surma, A. Miros, B. Piatkowski, E. Søndergård and B. Holst, New J. Phys. 12, 033018 (2010).

${ }^{11}$ D. Barredo, G. Laurent, F. Calleja, P. Nieto, J. J. Hinarejos, A. L. Vázquez de Parga, D. Farías, and R. Miranda, Appl. Phys. Lett. 96, 081901 (2010).

${ }^{12}$ D. Barredo, F. Calleja, P. Nieto, J. J. Hinarejos, G. Laurent, A. L. Vázquez de Parga, D. Farías, and R. Miranda, Adv. Mater. 20(18), 3492 (2008).

${ }^{13}$ B. Holst, private communication (2011).

${ }^{14}$ J. G. King and W. R. Bigas, Nature 222, 261 (1969).

${ }^{15}$ J. C. Weaver and J. G. King, Proc. Natl. Acad. Sci. U.S.A 70(10), 2781 (1973).

${ }^{16}$ J. Braun, P. K. Day, J. P. Toennies, G. Witte, and E. Neher, Rev. Sci. Instrum. 68(8), 3001 (1997).

${ }^{17}$ Atomic and Molecular Beam Methods, Edited by D. R. Miller and G. Scoles (Oxford University Press, New York, 1988), pp. 14-51.

${ }^{18}$ H. Pauly, Atom, Molecule, and Cluster Beams I (Springer, Berlin, 2000).

${ }^{19}$ M. P. Grams, A. M. Cook, J. H. Turner and R. B. Doak, J. Phys. D: Appl. Phys. 39, 930 (2006).

${ }^{20}$ T. Reisinger, G. Bracco, S. Rehbein, G. Schmahl, W. E. Ernst and B. Holst, J. Phys. Chem A 111(49), 12620 (2007).

${ }^{21}$ R. G. Livesey, Foundations of Vacuum Science and Technology, edited by J. M. Lafferty (Wiley Interscience, New York, 1998), pp. 81-137.

${ }^{22}$ D. J. Riley, M. Mann, D. A. MacLaren, P. C. Dastoor, and W. Allison, Nano Lett. 3(10), 1455 (2003).

${ }^{23}$ M. DeKieviet, D. Dubbers, M. Klein, U. Pieles, and C. Schmidt, Rev. Sci. Instrum. 71, 2015 (2000).

${ }^{24}$ R. B. Doak, J. Phys.: Condens. Matter 16(29), S2863 (2004).

${ }^{25}$ S. Maruyama, H. Kinbara, H. Hayashi, and D. Kimura, Microscale Thermophys. Eng., 1(1), 39 (1997).

${ }^{26} \mathrm{~S}$. Eder, T. Reisinger, B. Holst, and G. Bracco, in Proceedings of the 9th IEEE Conference on Nanotechnology, Genoa, 2009 (IEEE, Piscataway, 2009), p. 391-393. 